\title{
Cognitive tests aid in clinical differentiation of Alzheimer's disease versus Alzheimer's disease with Lewy body disease: Evidence from a pathological study
}

\section{Martina Azar ${ }^{1}$ | Silvia Chapman ${ }^{2}$ ～Yian Gu ${ }^{2}$ ～James B. Leverenz ${ }^{3}$ | Yaakov Stern ${ }^{2}$ Stephanie Cosentino ${ }^{2}$}

\author{
${ }^{1}$ Department of Psychology, Drexel \\ University, Philadelphia, Pennsylvania, USA \\ ${ }^{2}$ Cognitive Neuroscience Division, \\ Department of Neurology, Gertrude H. \\ Sergievsky Center, Taub Institute for Research \\ on Alzheimer's Disease and the Aging Brain, \\ Columbia University Medical Center, New \\ York, New York, USA \\ ${ }^{3}$ Lou Ruvo Center for Brain Health, \\ Neurological Institute, Cleveland Clinic \\ Foundation, Cleveland, OH, USA
}

\section{Correspondence}

Stephanie Cosentino, Columbia University Medical Center, 630 168th Street, P \& S Box 16, New York, NY 10032, USA.

Email:sc2460@cumc.columbia.edu

Funding information

NIA, Grant/Award Number: R01 AG007370

\begin{abstract}
Introduction: Clinical differentiation between Alzheimer's disease (AD) and AD with Lewy body disease (LBD) is relatively imprecise. The current study examined pathologically confirmed group differences in neuropsychological functioning, and the classification ability of specific tests.

Methods: Fifty-one participants with postmortem diagnoses of $A D(n=34)$ and $A D$ plus LBD ( $n=17)$ were drawn from the Predictors Study. One-way analyses of variance (ANOVAs) and $\chi^{2}$ analyses examined group differences in neuropsychological performance. Binary logistic regressions examined predictive utility of specific tests for pathological diagnosis.

Results: Individuals with AD had better visuoconstruction $(P=.006)$, phonemic fluency $(P=.08)$, and processing speed than AD plus $\operatorname{LBD}(P=.013)$. No differences were found in memory, naming, semantic fluency, or set-switching. Processing speed and visuoconstruction predicted pathologic group $(P=.03)$.

Discussion: Processing speed and visuoconstruction predicted postmortem diagnosis of $A D$ versus $A D$ plus LBD. Current results offer guidance in the selection and interpretation of neuropsychological tests to be used in the differential diagnosis of early dementia.
\end{abstract}

\section{KEYWORDS}

Alzheimer's disease, autopsy, diagnosis, Lewy body dementia, neuropsychological performance

\section{1 | BACKGROUND}

Alzheimer's disease (AD) is a progressive, dementing disease patholog-
ically characterized by plaques and neurofibrillary tangles beginning
in the temporal lobes, which eventually spread to the networks con-
necting the frontal, anterior, and parietal lobes. ${ }^{1,2}$ Lewy body disease
(LBD), another cause of dementia, is pathologically characterized by
abnormal accumulation of alpha synuclein in the brainstem as well
as diffusely throughout the cortex, with depleted neurotransmitters
such as dopamine and acetylcholine. In addition, it is common for Lewy bodies to spread to limbic areas. ${ }^{3}$ Although AD and LBD have unique pathological profiles, patients commonly present with pathologies characteristic of both diseases.. ${ }^{4,5}$ In fact, $\approx 50 \%$ of individuals with LBD have enough $A D$ pathology to be characterized as having a secondary diagnosis of $A D$, and vice versa. 6,7

However, the clinical differentiation between AD and AD plus LBD is relatively imprecise, as there is considerable overlap of cognitive symptoms and neuroanatomic substrates. ${ }^{8,9}$ In fact, although there are current criteria for clinical diagnosis of pure LBD, these criteria do not map well to AD plus LBD, the latter for which new diagnostic criteria 
THE JOURNAL OF THE ALZHEIMER'S ASSOCIATION

remain to be determined. ${ }^{7}$ Further characterization of differences, such as specific cognitive profiles, between $A D$ versus $A D$ plus LBD may help specify what aspects of the clinical presentation are key for the differentiation of these overlapping disorders.

The study of various cognitive abilities across patients with $A D$ and those diagnosed with LBD has revealed that visual-spatial, attentional, information processing, and executive deficits are often more severe in LBD cases, whereas memory deficits (particularly recognition memory) are more severe in $A D$, at least in the beginning stages of disease. ${ }^{9-11}$ Individuals with LBD may thus be expected to show a profile of relatively preserved memory storage and differentially impaired visuospatial abilities, phonemic fluency, processing speed, and executive abilities tasks relative to individuals with AD. ${ }^{10,12}$ The majority of the studies that have examined these cognitive differences though, have been based on clinical diagnoses. ${ }^{13-22}$ Using clinical diagnosis as the independent grouping variable can be inherently circular, however, because the same data used to predict group classification were utilized for classifying diagnostic group at the outset. Furthermore, the frequencies of pure LBD cases versus mixed $A D$ with Lewy bodies cannot be specified in clinical studies, thus it is not clear if results are driven by pure LBD pathology or by mixed pathology.

In contrast to clinically based diagnoses, studies based on pathologically confirmed diagnoses provide an objective marker against which to examine cognitive symptoms in each group. These studies are scarce, however, likely due to challenges associated with recruiting individuals for autopsy, and the significant length of time required to obtain pathologic specimens. ${ }^{10,11,23}$ Within pathological studies examining cognitive functioning across $A D$ versus $A D$ plus $L B D$, results suggest that the mixed group is more likely to have better performance in memory (recall and/or recognition), ${ }^{24,25}$ worse visuospatial abilities (pentagons or clock drawing), ${ }^{26-28}$ worse verbal fluency, ${ }^{27-29}$ worse processing speed, ${ }^{27,28}$ and worse attention. ${ }^{26}$ These results though are not consistent across all studies, with several studies observing no cognitive differences across groups. ${ }^{11,30}$ Additional studies based on pathological data are thus needed to further examine if cognitive symptoms differ as a function of underlying pathology, to ultimately aid in the clinical differentiation of $A D$ versus $A D$ plus $L B D$. It is important to note that identifying the earliest cognitive and clinical predictors of diagnosis is critical because targeted cholinergic therapies might be more effective when delivered earlier ${ }^{4}$ as well as identification of targeted treatment and functionally meaningful outcomes based on cognitive functioning that are tailored to each patient.

The current study examined group differences in performance across a range of neuropsychological tests including measures of visuoconstruction, processing speed, memory, language, attention, and executive functioning. Based on neuroanatomical and clinical evidence to date, it was hypothesized that the AD group would have higher visuoconstruction, phonemic fluency, executive functioning, and processing speed scores than the AD plus LBD group. The AD group was also expected to perform worse on memory (eg, recall, recognition) and semantic processing tasks (ie, naming, semantic fluency ratio) than those with mixed pathology. Finally, we examined the ability of neuropsychological tests that differed at the group level to classify

\section{RESEARCH IN CONTEXT}

1. Systematic review: The authors reviewed the literature using traditional (ie, PubMed) sources, including abstracts and presentations.

2. Interpretation: Our findings extend previous research on neuropsychological differences between pure Alzheimer's disease (AD) and mixed $A D$ plus $L B D$ (Lewy body disease), noting that the pure $A D$ and $A D$ plus LBD group differed in executive, processing speed, and visuoconstructional abilities but not in memory or semantic processing. However, only processing speed and visuoconstruction were adequate discriminants between pathologic groups.

3. Future Directions: The article highlights that processing speed and visuoconstruction may assist in the clinical differentiation of $A D$ versus $A D$ plus $L B D$, above and beyond the presence of visual hallucinations and extrapyramidal signs. In an effort to improve clinical decision-making and intervention earlier in the disease course, future work should examine whether qualitatively characterizing visuoconstructional dysfunction further improves its predictive utility and enhances that of classical LBD features.

individuals into pathologic group, while accounting for noncognitive symptoms (ie, behavioral, and extrapyramidal signs) that are often present in individuals with LBD.

\section{METHODS}

\section{1 | Participants}

The current sample was drawn from the Predictors 2 cohort's baseline visit, and comprised individuals whose autopsy data revealed the presence of $A D(n=34)$, or mixed AD plus $\operatorname{LBD}(n=17)$ with a Clinical Dementia Rating Scale score of 1 or 2. Participants were included in the current study if neuropsychological variables of interest and pathological diagnoses were available. The Predictors 2 cohort was initiated in 1997 following the same methods as the Predictors 1 cohort described previously. ${ }^{31}$ This cohort consisted primarily of individuals clinically diagnosed with $A D$, but also included an additional subset of patients with clinically diagnosed dementia with Lewy bodies (DLB) diagnosed according to the 1996 Consensus Guidelines for DLB. In the current sample, 32 individuals carried clinical diagnoses of AD, and 9 of DLB. Specific details of the general inclusion/exclusion criteria have been described previously. ${ }^{9}$ Only individuals from Predictors 2 cohort were included in the current study due to more specific neuropsychological and pathological data collected and thus available for the current study. 
In all, 211 subjects with probable AD, and 28 with probable LBD, were recruited into the cohort at three sites: Columbia University, Johns Hopkins University, and Massachusetts General Hospital. These individuals were diagnosed in the clinic and referred by their physicians to this study.

\subsection{Measures and procedure}

Neuropsychological measures included memory recall and recognition total (Hopkins Verbal Learning Test), naming (ie, Boston Naming Test), verbal fluency (Category fluency, CFL), processing speed (Trail Making Part A, TMT-A), executive functioning (Trail Making Part B, TMT-B), and pentagon copy from the (modified Mini-Mental State Exam, $\mathrm{mMMSE}^{32}$ ). In addition to examining performance on each test, we also examined the ratio of semantic to phonemic fluency (semantic/(semantic + phonemic), as it has been shown to be particularly specific to $A D{ }^{33}$ It reflects the relative contribution of semantic impairment to fluency deficits independent of defective retrieval that may occur secondary to frontal or subcortical compromise. Lower values represent fewer words generated for semantic versus phonemic fluency. Global cognitive (mMMSE) and functional impairment (Clinical Dementia Rating Scale, $C D R^{34}$ ) were measured for the study and used to determine whether groups were at a similar level of disease severity, but these were not primary outcomes of interest. Given the high relevance of non-cognitive symptoms in clinical classification of disease, specifically in LBD, visual hallucinations (coded dichotomously, 1 representing endorsement and 0 representing absence of symptoms), and parkinsonism scores were included in analyses. Parkinsonism was rated on a 5-point scale for each of the following: tremor at rest, rigidity in neck, limbs, and posture, and bradykinesia for a total possible sum score of 0 to 25 . Informants answered questions regarding medications and psychiatric and neurological history. The project was approved by the institutional review board at each of the three respective sites. All patients and their proxy decision-makers provided written informed consent. ${ }^{11}$

\subsection{Procedure of pathological diagnoses}

Cases were classified as having AD neuropathology if Braak Stage for neurofibrillary tangles was IV, V, or VI and CERAD neuritic plaque score was "moderate" or "frequent." Coexistent LBD was classified based on the presence of $\alpha$-synuclein immunohistochemistry positive inclusions consistent with "limbic" or "neocortical" Lewy body pathology. ${ }^{35,36}$

\subsection{Data analysis}

All analyses were conducted using SPSS 22. $\chi^{2}$ analyses and one-way analysis of variance (ANOVA) examined demographic and cognitive differences between the two autopsy-confirmed groups. Binary logistic regressions were used to determine the predictive value of individ- ual neuropsychological performance measures on pathological diagnosis post-mortem, and a single binary regression included multiple neuropsychological predictors established to be significant in the previous models; all models was also conducted while accounting for presence of non-cognitive symptoms (ie, visual hallucinations and parkinsonism). Pathological groups were coded $(A D=0$, mixed $A D$ plus $L B D=1)$. One individual with $C D R=0$ was removed from the analyses to ensure similar functional levels across participants. Individuals classified as having only LBD comprised a small sample $(n=12)$, hindering adequate group comparisons (ie, requiring greater power to detect differences across the three groups) and thus warranting lack of its inclusion in the study.

\section{3 | $\quad$ RESULTS}

\section{1 | Demographics}

The average global cognitive performance, as measured by the mMMS, was 35.95 ( $S D=7.55$ ). The average age ranged from 56 to 90 (mean $=74.71, \mathrm{SD}=8.38$ ) and education ranged from 8 to 20 years (mean $=14.59, \mathrm{SD}=3.22$ ). $49.2 \%$ were male, and $98.3 \%$ were white. Oneway ANOVAs and $\chi^{2}$ analyses revealed no differences in disease severity (CDR, mMMSE) or demographic variables across pathological diagnosis (Table 1). Clinical diagnoses of AD were accurate in $94.1 \%$ of the cases (32 out of 34). $5.9 \%$ of the cases (2 out of 34 ) with AD pathology were misclassified as DLB. Clinical diagnoses of DLB were accurate in $41.2 \%$ of cases (7 out of 17). $58.8 \%$ of the cases (10 out of 17) with mixed $A D$ and $L B D$ were misclassified as $A D$.

\subsection{Cognitive comparisons}

The AD group demonstrated higher phonemic fluency $F(1,41)=5.16$, $P=.029$ and faster processing speed $F(1,38)=5.72, P=.022$ than the $A D$ plus $L B D$ group. Pentagon copy was also less frequently impaired in AD (24\%; 8 of 33) than AD plus LDB (73\%; 11 of 15$), \chi^{2}(1)=10.49$, $P=.003$. All other cognitive measures were comparable across groups. For comparison of performance on the full neuropsychological battery (Table 2).

\subsection{Prediction of group membership}

Three binary logistic regression models examined the ability of each of the significant neuropsychological tests (CFL, Trails A, pentagons) to predict group membership. For model comparison purposes, participants were selected only if they had available data for all three measures, resulting in a sample of 39 patients ( $28 \mathrm{AD}$ and $11 \mathrm{AD}$ plus LBD). In addition, to enable more direct comparison of the predictors, CFL and TMT-A raw scores were converted to standardized scores adjusted for age and education using the same normative data set. ${ }^{37,38}$ Normative data were not available for pentagon copy. 
THE JOURNAL OF THE ALZHEIMER'S ASSOCIATION

TAB LE 1 Demographics and clinical characteristics of sample

\begin{tabular}{|c|c|c|c|c|}
\hline & Pure AD $(n=34)$ & Mixed AD plus LBD $(n=17)$ & $F$ & $P$ \\
\hline Gender (\% female) & 52.9 & 64.7 & $\chi^{2}=0.65$ & .31 \\
\hline Ethnicity (\% Caucasian) & 100 & 94.1 & $\chi^{2}=2.20$ & .34 \\
\hline Education & $14.62(3.04)$ & $15.53(2.76)$ & 1.08 & .30 \\
\hline mMMSE total score & $21.15(3.58)$ & $19.07(5.04)$ & 2.70 & .11 \\
\hline Time from assessment to death & $1888.33(861.41)$ & $1906.00(1219.67)$ & 0.01 & .96 \\
\hline
\end{tabular}

AD, Alzheimer's disease; CDR, Clinical Dementia Rating; LBD, Lewy body disease; mMMSE, modified Mini-Mental State Exam.

TABLE 2 Cognitive scores (mean and SD)

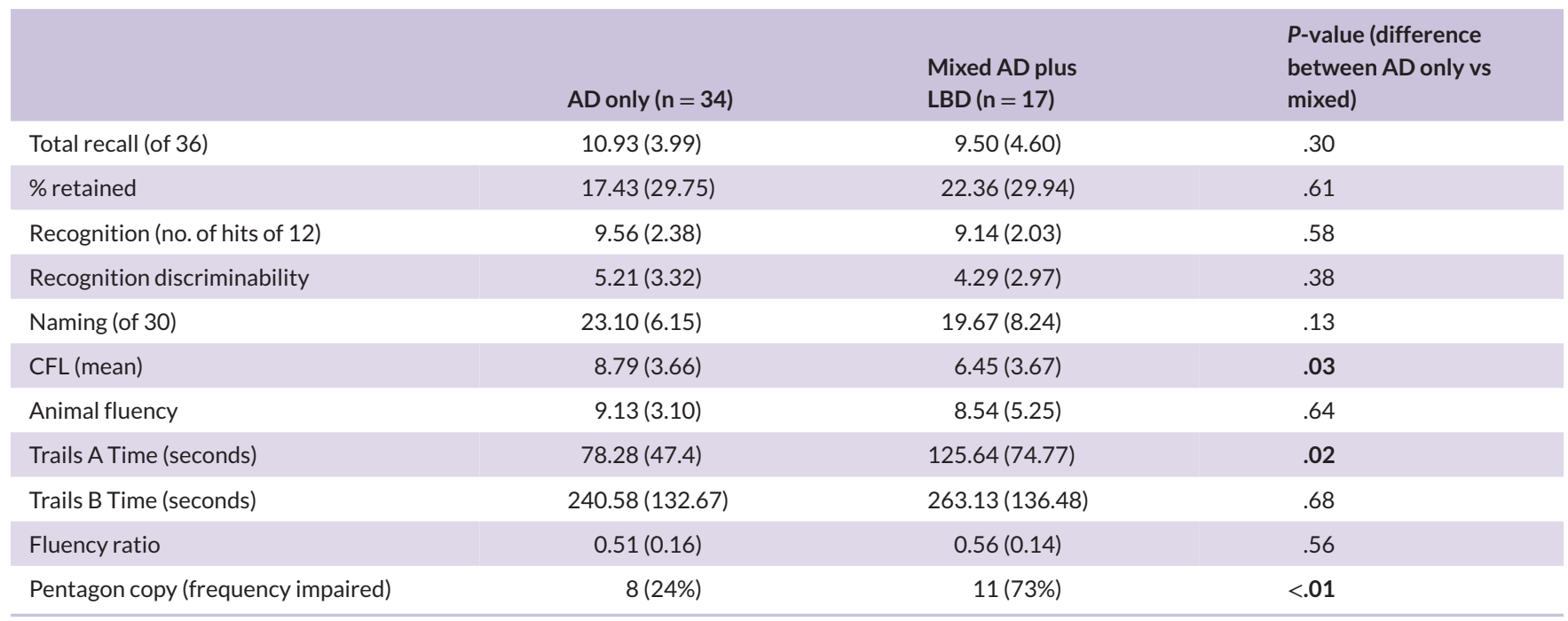

Note: Significant values are bolded and defined by $P<.05$.

AD, Alzheimer's disease; CFL, category fluency; LBD, Lewy body disease.

Standardized CFL score was marginally predictive of group $(b=$ -0.18 , standard error $[\mathrm{SE}]=0.11$, odds ratio $=0.86, P=.09,95 \%$ $\mathrm{Cl}[0.69,1.03]), \chi^{2}(1)=3.10, P=.08$. The Hosmer-Lemeshow test of homogeneity was not significant $\chi^{2}(7)=7.92, P=.34$. CFL correctly classified $76.9 \%$ of participants $(96.4 \%$ of the AD group, $27.3 \%$ of mixed $A D$ plus $L B D$ ), explaining a relatively small proportion of the variance in diagnosis classification, Cox and Snell $R^{2}=0.08$, Nagelkerke $R^{2}=0.11$. Figure 1 shows the area under the receiver-operating characteristic (ROC) curve $=0.67(95 \% \mathrm{Cl} 0.48$ to 0.86 ), reflecting poor discrimination according to Hosmer et al. ${ }^{39}$ Once the same model was run with inclusion of behavioral and extrapyramidal factors (ie, visual hallucinations and parkinsonism), the results continued to hold, with CFL score predicting group classification $(b=-0.21, \mathrm{SE}=0.10$, odds ratio $=0.82, P=.03,95 \% \mathrm{Cl} 0.67$ to $\left.1.00, \chi^{2}(3)=7.99, P=.05\right)$. CFL correctly classified $78.9 \%$ of participants ( $92.3 \%$ of the AD group, $45.5 \%$ of the mixed AD plus LBD group), explaining a relatively small proportion of the variance in diagnosis classification, Cox and Snell $\mathrm{R}^{2}=0.19$.

TAB LE 3 Non-cognitive scores (sum of symptoms; mean and SD)

\begin{tabular}{|c|c|c|c|}
\hline & AD only $(n=34)$ & $\begin{array}{l}\text { Mixed AD plus } \\
\text { LBD }(n=17)\end{array}$ & $\begin{array}{l}P \text {-value (difference } \\
\text { between } A D \text { only vs } \\
\text { mixed) }\end{array}$ \\
\hline Visual hallucinations & $0.09(0.38)$ & $0.71(1.05)$ & .004 \\
\hline
\end{tabular}

AD, Alzheimer's disease; LBD, Lewy body disease. 


\section{ROC Curve}

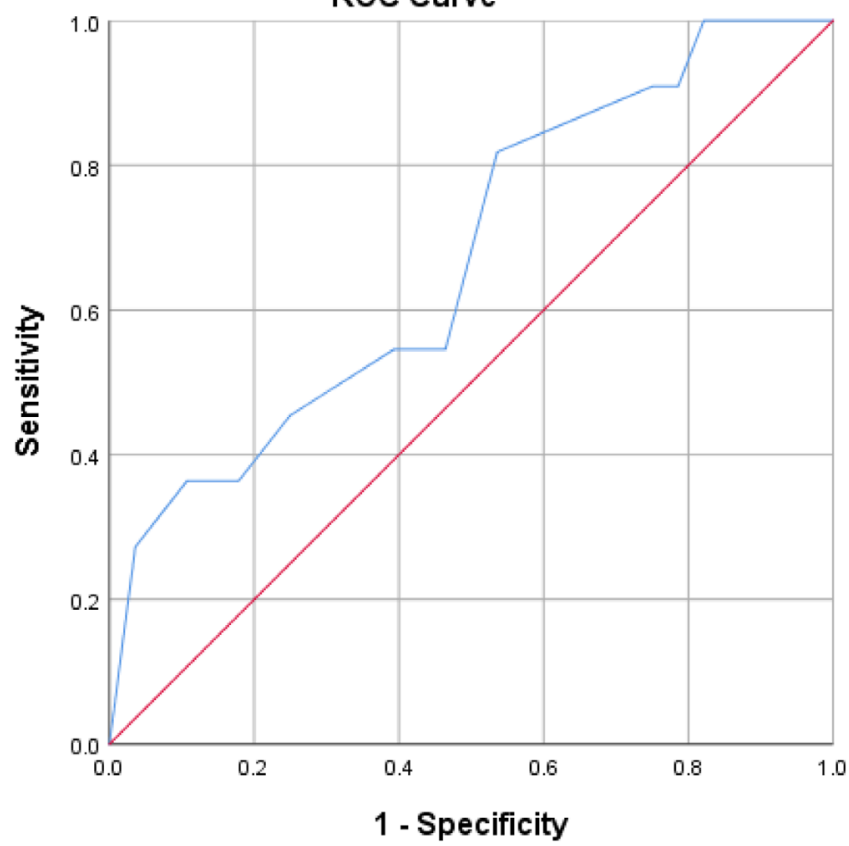

FIGURE 1 ROC curve of phonemic fluency

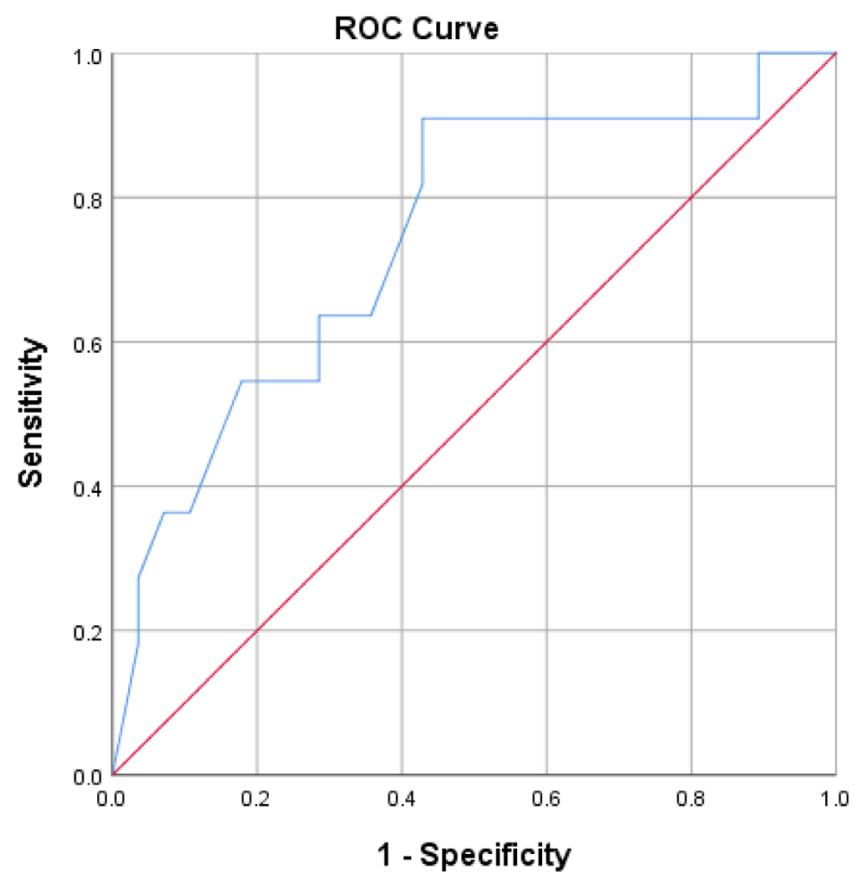

FIGURE 2 ROC curve of processing speed measure

Standardized TMT-A score was significantly predictive of group classification with the odds of being classified as mixed pathology increasing as time to complete TMT-A increases $(b=-0.30, S E=0.14$, odds ratio $=0.74, P=.03,95 \% \mathrm{Cl} 0.57$ to 0.98$), \chi^{2}(1)=6.13, P=.013$. The Hosmer-Lemeshow test of homogeneity was not significant $\chi^{2}(8)$ $=7.95, P=.44$. This second model correctly classified $76.9 \%$ of participants ( $92.9 \%$ of $A D$ and $36.4 \%$ of $A D$ plus $L B D)$. Figure 2 shows the area under the ROC curve as $0.75(95 \% \mathrm{Cl}, 0.57$ to 0.92$)$, having acceptable

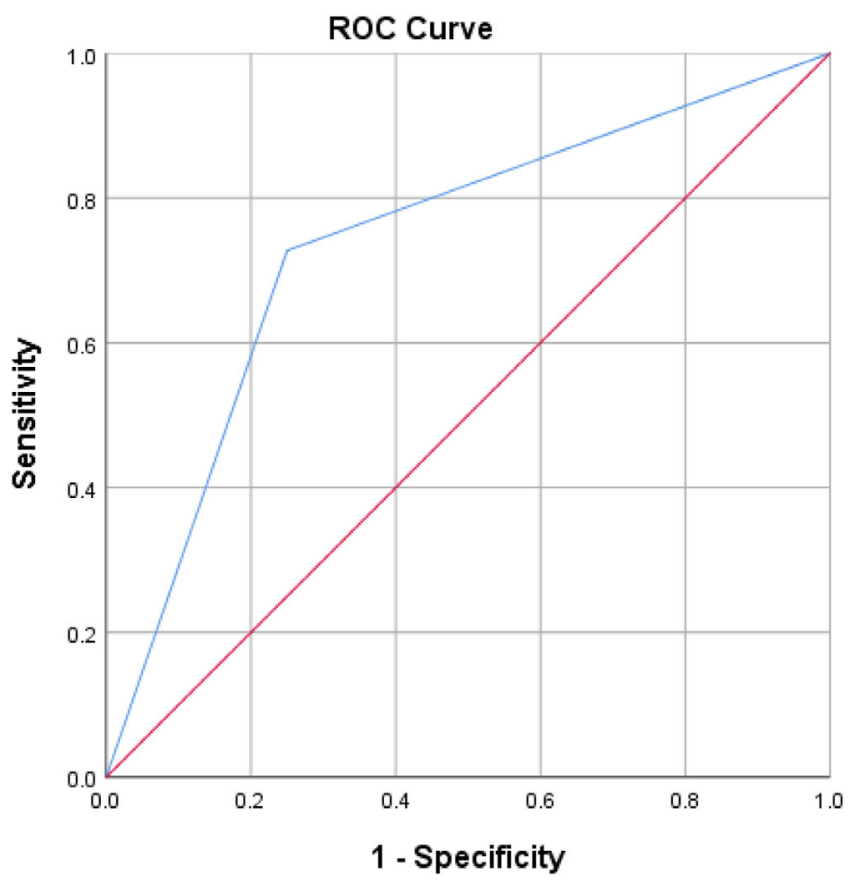

FIGURE 3 ROC curve of visuoconstruction

discrimination according to Hosmer et al. ${ }^{39}$ Once again, this model was conducted with inclusion of non-cognitive factors (ie, visual hallucinations and parkinsonism). The findings reflected that TMT-A was significantly predictive of group classification, above and beyond presence of visual hallucinations and extrapyramidal signs $(b=0.02, \mathrm{SE}=0.01$, odds ratio $=1.02, P=.05,95 \% \mathrm{Cl} 1.00$ to $\left.1.03, \chi^{2}(3)=6.87, P=.07\right)$.

Pentagon copy significantly contributed to group classification (b $=2.08, \mathrm{SE}=0.81, P=.01$, odds ratio $=8.00,95 \% \mathrm{Cl} 1.65$ to 38.79 ), $\chi^{2}(1)=7.59, P=.006$, and explained $\approx 20 \%$ of the variance (Cox and Snell $R^{2}=0.18$, Nagelkerke $R^{2}=0.25$ ). The model classified $74.4 \%$ of cases correctly ( $75 \%$ pure $A D$ and $72.7 \%$ AD plus LBD). Figure 3 shows the area under the curve (AUC) $=0.74$ ( $95 \% \mathrm{Cl} 0.56$ to 0.092), having acceptable discrimination. ${ }^{39}$ Once this model was conducted taking into consideration visual hallucinations and parkinsonism symptoms, visuoconstruction (per the pentagon copy score) remained a significant predictor over pathological group classification $(b=-2.60, \mathrm{SE}=$ 0.97 , odds ratio $=0.08, P=.01,95 \% \mathrm{Cl} 0.01$ to $0.49, \chi^{2}(3)=11.20$, $P=.01$ ).

A final model included TMT-A and pentagon copy; both deemed to have acceptable discrimination. Although this model significantly predicted diagnosis, $\chi^{2}(3)=8.95, P=.03$, neither predictor remained an independently significant group classifier (TMTA, $b=-0.16, \mathrm{SE}=0.18$, $P=.39,95 \% \mathrm{Cl} 0.60$ to 1.22 ; pentagons, $\mathrm{b}=-1.47, \mathrm{SE}=0.93, P=.23$, $95 \% \mathrm{Cl} 0.04$ to 1.44$)$. Furthermore, combining these predictors overall predicted $76.9 \%$ of cases (85.7\% correctly classified AD and $54.5 \%$ correctly classified AD plus LBD) and not improve overall classification above that of TMT-A alone (76.9\%) or pentagon copy (74.4\%). Figure 4 depicts the AUC as 0.78 ( $95 \% \mathrm{Cl} 0.61$ to 0.95 ), reflecting an acceptable discrimination. ${ }^{39}$ 
THE JOURNAL OF THE ALZHEIMER'S ASSOCIATION

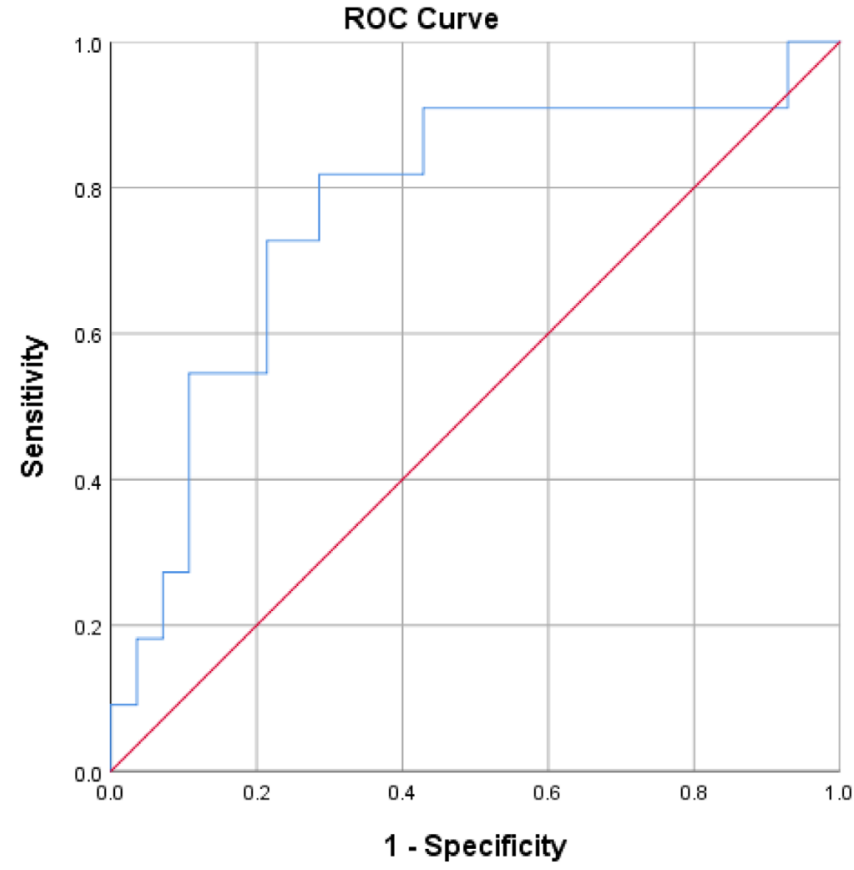

FIGURE 4 ROC curve of processing speed and visuoconstruction measures

\section{DISCUSSION}

The current study sought to further the understanding of neuropsychological differences across pathologically confirmed cases of $A D$ versus $A D$ plus $L B D$, and to identify cognitive tests that may aid the clinical differentiation of these overlapping diseases. Previous research has indicated that certain cognitive functions may help discriminate these groups, such that memory storage may be relatively spared in LBD in contrast to greater impairments in visuospatial function, executive function, processing speed, and attention, although findings have not been consistent. ${ }^{11,24,30}$ Results from the current study indicated that the $A D$ and $A D$ plus LBD groups differed on assessments of phonemic fluency (CFL), processing speed (Trails A), and visuoconstruction (pentagon copy). As will be discussed below, each of these tests had different utility for classifying participants into the correct pathological group.

Findings from the current study are generally in line with those previous pathological studies comparing $A D$ versus $A D$ plus $L B D$, particularly those showing that the latter group is more likely to be impaired in phonemic fluency and other measures of executive functioning than pure AD. ${ }^{27,29}$ These results are not consistent across all studies, however Yoshizawa et al. ${ }^{11}$, potentially reflecting sampling differences. For example, Yoshizawa et al. ${ }^{40}$ included patients in a somewhat earlier stage of dementia than the current study (eg, CDR ranging from 0 to 1 ). It is possible that differences in executive functioning had not emerged yet across groups in that study. However, the ROC curve for fluency in the current study indicated that it was a poor discriminator of diagnosis; it correctly classified over $70 \%$ of the sample, but this was driven mainly by correct classification of $A D(>90 \%)$ and generally poor clas- sification of AD plus LBD ( $<30 \%$ ). Phonemic fluency alone, therefore, may not represent a very reliable determinant of the presence of $L B D$.

Our results also showed that participants with AD plus LBD had slower processing speed than those with AD. This result, to our knowledge, has been reflected only in two other studies. ${ }^{27,28}$ In the current study, the processing speed measure (TMT-A) correctly classified the same percentage of overall patients as the phonemic fluency measure (77\%), and its classification of AD plus LBD was only slightly more accurate (36\%). It is interesting that Trail Making Part B (TMT-B), a measure that relies on both speed and executive function, did not differ across groups. It may be that the slowing associated with DLB differentially impairs performance on TMT-A, a relatively simple measure of attention and speed on which patients with mild AD can perform well. However, on TMT-B, the executive set-shifting component may lower performance in both groups to a similar enough degree that statistically meaningful differences do not emerge across groups. We also considered the possibility that a floor effect on TMT-B could have reduced a potential difference across groups; however, inspection of data (not shown) is not consistent with this idea.

Like phonemic fluency and processing speed, visuoconstruction (pentagon copy) was more likely to be impaired in AD plus LBD than in $A D$ alone, and this measure was deemed to have acceptable discrimination ability, ${ }^{39}$ correctly classifying a total of $74 \%$ of patients, above and beyond the contribution of behavioral and extrapyramidal factors often presenting with LBD symptomatology. This finding is in line with those of other studies that have reported worse visuospatial functioning (eg, pentagons, clock drawing) in AD plus LBD than in $A D$ alone. ${ }^{26-28,41}$ Of interest, of the three neuropsychological measures that differed across groups, pentagon copy seemed to be most sensitive to the presence of comorbid LBD pathology, correctly classifying $73 \%$ of this specific group (vs $27 \%$ and $36 \%$ for the other two measures). In turn, however, the pentagon copy misclassified more AD cases (25\%) than the other measures (4\% to $7 \%$ ). A caveat of this model is the dichotomous nature of the pentagon task, which limits its ability capture a broad range of visuoconstruction and visuospatial ability, and hence likely limits its predictive ability. A more fine-grained or qualitative evaluation of such abilities may detect more subtle differences between groups and improve group classification. Indeed, future studies with more comprehensive neuropsychological assessments, a more nuanced visuospatial measure, and attention to the qualitative examination of errors are needed to further elucidate how specific or combined cognitive abilities can contribute to the correct classification of disease.

Current results demonstrate the trade-off between the sensitivity and specificity of each measure for the identification of different pathologies, raising the question of whether combining the measures may yield the highest predictive accuracy. A final regression was thus conducted to determine whether combining the two best predictors of diagnosis (eg, processing speed and visuoconstruction, both of which were deemed to have acceptable discrimination) improved overall group classification. Of interest, the final model did not improve classification or AUC over that of processing speed or visuoconstruction alone. 
Despite the expectation that the pure AD group would show greater depletion in memory and retention given previous work demonstrating that recognition discriminability, is more impaired in $A D$ than in $A D$ plus DLB, ${ }^{4}$ and that hippocampal volume is relatively better preserved in LBD than $A D,{ }^{42}$ no significant differences were observed in memory (ie, retention and recognition) or in semantic processing abilities generally associated with temporal lobe functioning (ie, naming, semantic fluency, or the fluency ratio calculated to reflect semantic degradation specifically). The reason for similar levels of memory and semantic abilities in the current study, versus studies that compare AD to pure LBD in particular, may well be that both groups have AD pathology and are at similar levels of global cognitive impairment. It is also possible that heterogeneity in the regional distribution of both $A D$ and $D L B$ pathologies contributes to differences seen across studies. Specifically, it is possible that in the current sample, there could have been a relatively low burden of temporal pathology in the $A D$ group, or a relatively high burden of temporal pathology in the AD plus DLB group, with either scenario leading to comparable rather than dissimilar memory and language abilities. Indeed, AD can present with greater frontal involvement ${ }^{24}$ or, conversely, with disproportionate posterior burden as is seen in posterior cortical atrophy (PCA). In such cases, memory can be less affected than in the classic amnestic presentation of $A D$. In order to produce more reliable findings across studies, it will be important not only to compare pathological diagnosis in a dichotomous manner, but with consideration for the degree and distribution of each pathology.

This study supports the idea that cognitive testing can aid in the clinical differentiation between autopsy-confirmed $A D$ and $A D$ plus DLB, a common and important differential diagnosis that is not well differentiated when individuals come into the clinic for testing. In particular, processing speed and to a lesser extent visuoconstruction predicted pathological diagnosis with acceptable discrimination, and may assist in the clinical differentiation of these groups, above and beyond consideration of behavioral and extrapyramidal symptoms. It is worth noting, however, that although models had acceptable statistical AUC discrimination values, higher AUC (closer to one) represents the highest degree of both sensitivity and specificity. Results in this study inform the manner in which neuropsychological testing aids diagnostic classification, but should be considered in a broader context when applying to patient care, and in light of risks and benefits associated with the diagnostic process. Examination of classical LBD features such as extrapyramidal and psychiatric features other than parkinsonism and visual hallucinations (see ref 7 for current diagnostic criteria) should be examined in conjunction with these cognitive abilities to further understand the profile of symptoms, which taken together best discriminate between $A D$ versus AD plus LBD. Certainly, amyloid imaging studies, DaTScan and single-photon emission computed tomography (SPECT) scans (123I-FP-CIT SPECT ${ }^{43-45}$ ) can aid in detecting specific pathologies. For example, one previous study showed that cortical pittsburgh compound $B(\mathrm{PiB})$ represents retention differentiated patients with pathologically confirmed $A D(L B D+A D$ and $A D)$ from those with pure LBD with $93 \%$ accuracy, and the regional pattern of $A \beta$ spared the occipital lobes when LBD was present, regardless of whether $A D$ was also present. ${ }^{46}$ However, identifying the mixed presentation of $A D$ plus LBD would likely require multiple costly and invasive scans, and may not always be feasible. As such, determining the extent to which non-invasive, routine, and inexpensive neuropsychological measures inform differential diagnosis is an important endeavor.

Regarding the clinical implications of the current findings, it is worth noting that clinicians would benefit from accurate differentiation between diagnosis to provide adequately tailored recommendations and refer patients to specific services that educate and target symptoms common in either diagnosis (ie, AD, LBD, mixed AD plus LBD). Accurate diagnosis is crucial to help in prognosis, medical decisionmaking, education on treatment options, and treatments to alleviate symptoms. Specifically, this distinction can allow individuals to receive appropriate services and obtain cognitive enhancing medications for the specific diagnosis (ie, cholinergic therapy), and better educate patients and their families on the expected timeline of progression of and extent of symptoms. Furthermore, tailored clinical recommendations can be directed toward comorbid symptoms that are more commonly present in specific or combined diagnoses (ie, depression in $A D$, or hallucinations, sleep difficulty, and extrapyramidal features in LBD) and improvement of quality of life (ie, community resources, support groups). As services recommended on a neuropsychological evaluation often depend on the extent, type, and etiology of deficit, clinical differentiation is essential. As such, clinical differentiation is key for addressing tailored functionally meaningful outcomes for each patient. Visuospatial and processing speed difficulties often observed in LBD may directly influence driving ability and thus would warrant a driving evaluation once these deficits are clinically established. Executive deficits, including attention, working memory, and set-switching may influence decision-making and adherence to recommendations provided by respective medical providers (ie, medication management). Thus, recommendations to attain specific services and educate caregivers and patients can only occur once these deficits are identified, preferably early in the disease process. Not only do the current findings point to aspects of the neuropsychological profile that can inform differential diagnosis if subtle or questionable signs of LBD are present along with cognitive impairment, but when comprehensive neuropsychological assessment is not available, brief assessments of processing speed and pentagon copy may inform the presence of LBD.

There are several limitations of the current study. Regarding the clinical characterization of participants, it is possible that individuals developed motor symptoms after the current study visit. Although we cannot account for this in the current study, it lends for future longitudinal research to establish a timeline of cognitive and non-cognitive (ie, extrapyramidal, behavioral) symptoms that account for changes in clinical profile throughout the respective disease process. Another limitation of the current study included disparate and relatively small sample sizes. Due to this limitation, we were unable to include a pure LBD group in the current study; this group could have informed the extent to which differences in test performance were due solely to the presence of LBD pathology or represented an interaction between mixed pathologies. Nonetheless, the current study sought to focus on those individuals that are often difficult to diagnose (ie, $A D$ with $L B D$ ) from those relatively easier to classify (ie, pure $A D$ ) and thus fill a greater 
gap in the literature. This limitation reflects the challenges associated with recruiting individuals for autopsy, as previously mentioned, and should be improved in future research. Regarding analyses, this study did not conduct a Bonferroni correction of the initial between group analyses examining neuropsychological measures, which allowed CFL and Trail Making Test A to remain significant and thus be included in subsequent classification models. However, given that the sample consisted of cases with CDR 1 and 2, subtle cognitive differences were expected between the groups and providing such a stringent correction might have led to increased likelihood of type 2 error. In addition, it is possible that more complex analytical methods such as machine learning (ie, Random forest model) may have allowed for interesting and more nuanced observations of interactions of multivariate data presented in the current study. This represents an interesting area of future research.

\section{ACKNOWLEDGMENTS}

The authors would like to thank the National Institute on Aging (NIA) for funding this research (R01 AG007370).

\section{REFERENCES}

1. Serrano-Pozo A, Frosch MP, Masliah E, Hyman BT. Neuropathological alterations in Alzheimer disease. Cold Spring Harb Perspect Med. 2011;1(1):a006189.

2. Kreisl WC, Lyoo CH, Liow J.-S, et al. Distinct patterns of increased translocator protein in posterior cortical atrophy and amnestic Alzheimer's disease. Neurobiol Aging. 2017;51:132-140.

3. Dickson DW. Dementia with Lewy bodies: neuropathology. J Geriatr Psychiatry Neurol. 2002;15(4):210-216.

4. Hamilton JM, Salmon DP, Galasko D, et al. A comparison of episodic memory deficits in neuropathologically-confirmed Dementia with Lewy bodies and Alzheimer's disease. J Int Neuropsychol Soc 2004;10(5):689-697.

5. Hansen LA, Samuel W. Criteria for Alzheimer's disease and the nosology of dementia with Lewy bodies. Neurology. 1997;48(1):126-132.

6. Irwin DJ, Hurtig HI. The Contribution of tau, amyloid-beta and alphasynuclein pathology to dementia in Lewy body disorders. J Alzheimers Dis Parkinsonism. 2018;8(4):444.

7. McKeith IG, Boeve BF, Dickson DW, et al. Diagnosis and management of dementia with Lewy bodies: fourth consensus report of the DLB Consortium. Neurology. 2017;89(1):88-100.

8. Tiraboschi P, Salmon DP, Hansen LA, Hofstetter RC, Thal LJ, CoreyBloom J. What best differentiates Lewy body from Alzheimer's disease in early-stage dementia? Brain. 2006;129(Pt 3):729-735.

9. Stavitsky K, Brickman AM, Scarmeas N, et al. The progression of cognition, psychiatric symptoms, and functional abilities in dementia with Lewy bodies and Alzheimer disease. Arch Neurol. 2006;63(10):14501456.

10. Metzler-Baddeley C. A review of cognitive impairments in dementia with Lewy bodies relative to Alzheimer's disease and Parkinson's disease with dementia. Cortex. 2007;43(5):583-600.

11. Yoshizawa H, Vonsattel JP, Honig LS. Early neuropsychological discriminants for Lewy body disease: an autopsy series. J Neurol Neurosurg Psychiatry. 2013;84(12):1326-1330.

12. Collerton D, Burn D, McKeith I, O'Brien J. Systematic review and meta-analysis show that dementia with Lewy bodies is a visualperceptual and attentional-executive dementia. Dement Geriatr Cogn Disord. 2003;16(4):229-237.

13. Gnanalingham KK, Byrne EJ, Thornton A, Sambrook MA, Bannister $P$. Motor and cognitive function in Lewy body dementia: comparison with Alzheimer's and Parkinson's diseases. J Neurol Neurosurg Psychiatry. 1997;62(3):243-252

14. Walker Z, Allen RL, Shergill S, Katona CL. Neuropsychological performance in Lewy body dementia and Alzheimer's disease. Br J Psychiatry. 1997;170:156-158.

15. Mori E, Shimomura T, Fujimori M, et al. Visuoperceptual impairment in dementia with Lewy bodies. Arch Neurol. 2000;57(4):489-493.

16. Lambon Ralph MA, Powell J, Howard D, Whitworth AB, Garrard P, Hodges JR. Semantic memory is impaired in both dementia with Lewy bodies and dementia of Alzheimer's type: a comparative neuropsychological study and literature review. J Neurol Neurosurg Psychiatry. 2001;70(2):149-156

17. Calderon J, Perry RJ, Erzinclioglu SW, Berrios GE, Dening TR, Hodges JR. Perception, attention, and working memory are disproportionately impaired in dementia with Lewy bodies compared with Alzheimer's disease. J Neurol Neurosurg Psychiatry. 2001;70(2):157-164.

18. Ballard C, O'Brien J, Gray A, et al. Attention and fluctuating attention in patients with dementia with Lewy bodies and Alzheimer disease. Arch Neurol. 2001;58(6):977-982.

19. Doubleday E, Snowden J, Varma A, Neary D. Qualitative performance characteristics differentiate dementia with Lewy bodies and Alzheimer's disease. J Neurol Neurosurg Psychiatry. 2002;72(5):602607

20. Simard, M, van Reekum R, Myran D. Visuospatial impairment in dementia with Lewy bodies and Alzheimer's disease: a process analysis approach. Int J Geriatr Psychiatry. 2003;18(5):387-391.

21. Cormack F, Aarsland D, Ballard C, Tovée MJ. Pentagon drawing and neuropsychological performance in dementia with Lewy Bodies, Alzheimer's disease, Parkinson's disease and Parkinson's disease with dementia. Int J Geriatr Psychiatry. 2004;19(4):371-377.

22. Perriol M.-P, Dujardin K, Derambure $P$, et al. Disturbance of sensory filtering in dementia with Lewy bodies: comparison with Parkinson's disease dementia and Alzheimer's disease. J Neurol Neurosurgery Psychiatry. 2005;76(1):106-108

23. Breitve $\mathrm{MH}$, Chwiszczuk LJ, Hynninen MJ, et al. A systematic review of cognitive decline in dementia with Lewy bodies versus Alzheimer's disease. Alzheimers Res Ther. 2014;6(5-8):53.

24. Connor DJ, Salmon DP, Sandy TJ, Galasko D, Hansen LA, Thal LJ. Cognitive profiles of autopsy-confirmed Lewy body variant vs pure Alzheimer disease. Arch Neurol. 1998;55(7):994-1000.

25. Johnson, DK, Morris JC, Galvin JE. Verbal and visuospatial deficits in dementia with Lewy bodies. Neurology. 2005;65(8):1232-1238.

26. Ala TA, Hughes LF, Kyrouac GA, Ghobrial MW, Elble RJ. The MiniMental State exam may help in the differentiation of dementia with Lewy bodies and Alzheimer's disease. Int J Geriatr Psychiatry. 2002;17(6):503-509.

27. Hansen L, Salmon D, Galasko D, et al. The Lewy body variant of Alzheimer's disease. A clinical and pathologic entity. Neurology. 1990;40(1):1-8.

28. Galasko D, Katzman R, Salmon DP, Hansen L. Clinical and neuropathological findings in Lewy body dementias. Brain Cogn. 1996;31(2):166175.

29. Jicha GA, Schmitt FA, Abner E, et al. Prodromal clinical manifestations of neuropathologically confirmed Lewy body disease. Neurobiol Aging. 2010;31(10):1805-1813.

30. Förstl H, Burns A, Luthert P, Cairns N, Levy R. The Lewy-body variant of Alzheimer's disease. Clinical and pathological findings. Br J Psychiatry. 1993;162:385-392.

31. Stern $\mathrm{Y}$, Folstein M, Albert M, et al. Multicenter study of predictors of disease course in Alzheimer disease (the "predictors study"). I. Study design, cohort description, and intersite comparisons. Alzheimer Dis Assoc Disord. 1993;7(1):3-21.

32. Folstein, MF, Folstein SE, McHugh PR. "Mini-mental state". A practical method for grading the cognitive state of patients for the clinician. J Psychiatr Res. 1975;12(3):189-198. 
33. Rascovsky K, Salmon DP, Hansen LA, Thal LJ, Galasko D. Disparate letter and semantic category fluency deficits in autopsy-confirmed frontotemporal dementia and Alzheimer's disease. Neuropsychology. 2007;21(1):20-30.

34. Hughes CP, Berg L, Danziger WL, Coben LA, Martin RL. A new clinical scale for the staging of dementia. Br J Psychiatry. 1982;140:566-572.

35. Pillai JA, Butler RS, Bonner-Jackson A, Leverenz JB. Impact of Alzheimer's disease, Lewy body and vascular co-pathologies on clinical transition to dementia in a national autopsy cohort. Dement Geriatr Cogn Disord. 2016;42(1-2):106-116.

36. Tulloch J, Leong L, Chen S, et al. APOE DNA methylation is altered in Lewy body dementia. Alzheimers Dement. 2018;14(7):889-894.

37. Ivnik RJ, Malec JF, Smith GE, Tangalos EG, Petersen RC. Neuropsychological tests' norms above age 55: COWAT, BNT, MAE token, WRAT-R reading, AMNART, STROOP, TMT, and JLO. Clin Neuropsychol. 1996;10(3):262-278.

38. Strauss, E, Sherman EMS, Spreen O. A Compendium of Neuropsychological Tests: Administration, norms, and commentary. 3rd ed. New York: Oxford University Press; 2006.

39. Hosmer, DWJ, Lemeshow S, Sturdivant RX. Applied Logistic Regression. 3rd ed. : John Wiley and Sons, Inc.; 2013.

40. Yoshizawa, H, Vonsattel JPG, Honig LS. Early neuropsychological discriminants for Lewy body disease: an autopsy series. J Neurol Neurosurg Psychiatry. 2013;84(12):1326-1330.

41. Ala TA, Hughes LF, Kyrouac GA, Ghobrial MW, Elble RJ. Pentagon copying is more impaired in dementia with Lewy bodies than in Alzheimer's disease. J Neurol Neurosurg Psychiatry 2001;70(4):483488.
42. Kantarci K, Lesnick T, Ferman TJ, et al. Hippocampal volumes predict risk of dementia with Lewy bodies in mild cognitive impairment. Neurology. 2016;87(22):2317-2323.

43. Rioboo PJ, Varela Lema L, Serena Puig, A, Ruano-Ravina, A. Effectiveness of 123I-ioflupane (DaTSCAN) in the diagnosis of Parkinsonian syndromes. A systematic review. Rev Esp Med Nucl. 2007;26(6):375384.

44. McCleery J, Morgan S, Bradley KM, Noel-Storr AH, Ansorge O, Hyde C. Dopamine transporter imaging for the diagnosis of dementia with Lewy bodies. Cochrane Database Syst Rev. 2015;1:CD010633.

45. Vaamonde-Gamo, J, Flores-Barragan, JM, Ibanez, R, Gudin, M, Hernandez, A. DaT-SCAN SPECT in the differential diagnosis of dementia with Lewy bodies and Alzheimer's disease. Rev Neurol. 2005;41(11):276279.

46. Landau SM, Villemagne VL. Can amyloid PET differentiate "pure" LBD from AD with or without LBD copathology. Neurology. 2020;94(3):103104.

How to cite this article: Azar M, Chapman S, Gu Y, Leverenz J, Stern Y, Cosentino S. Cognitive tests aid in clinical differentiation of Alzheimer's disease versus Alzheimer's disease with Lewy body disease: Evidence from a pathological study. Alzheimer's Dement. 2020;1-9.

https://doi.org/10.1002/alz.12120 